\title{
Collective Interatomic Decay of Multiple Vacancies in Clusters
}

\author{
Vitali Averbukh ${ }^{1, *}$ and Přemysl Kolorenč ${ }^{2, \dagger}$ \\ ${ }^{1}$ Max Planck Institute for the Physics of Complex Systems, Nöthnitzer Str. 38, D-01187 Dresden, Germany \\ ${ }^{2}$ Institute of Theoretical Physics, Faculty of Mathematics and Physics, Charles University in Prague, \\ V Holešovičkách 2, 18000 Prague, Czech Republic \\ (Received 9 July 2009; published 27 October 2009)
}

\begin{abstract}
We predict that inner-shell ionization of more than one atom (or molecule) in a cluster, e.g., by intense free-electron laser radiation, can lead to an interatomic decay process in which the formed vacancy states decay simultaneously, while a neighboring neutral species is ionized. This collective decay phenomenon can be regarded as a transfer of two or more virtual photons from the ionized cluster units to a neutral one. Simulations of collective decay in $\left(4 s^{-1}, 4 s^{-1}\right)\left(\mathrm{Kr}^{+}\right)_{2} \mathrm{Ar}$ show that the two-virtual-photon process can be competitive with the dissociative nuclear dynamics of the doubly ionized cluster. Generality of the collective interatomic decay is discussed.
\end{abstract}

PACS numbers: $32.80 . \mathrm{Aa}, 31.15 . \mathrm{A}-$, 32.80.Zb, 36.40.-c

The advent of powerful free-electron lasers (FELs) operating in the UV and $x$-ray domains [1] is bringing the study of radiation-matter interaction to the new dynamical regime characterized both by high photon energies and high photon fluxes. When incident on atoms, molecules, or clusters, the strong fluxes of high-energy FEL photons lead to multiple inner-shell ionization (see, e.g., Ref. [2]). Subsequent decay of the created inner-shell vacancies, e.g., Auger decay [3], is accompanied by electron emission and thus contributes directly to the accumulation of positive charge in the target system and eventually to its decomposition by Coulombic explosion. Recently, the subject of the radiation damage caused by the x-ray FELs (XFELs) have come into focus of attention due to the intriguing possibility of single-molecule $\mathrm{x}$-ray diffraction in the gas phase [4]. A detailed understanding of electronic decay processes occurring in multiply inner-shell-ionized systems would certainly contribute to the emerging picture of coupled electronic and nuclear dynamics resulting in the radiation damage. In the present work, we would like to ask the following question: "Are there any electronic decay processes that occur exclusively in multiply inner-shellionized polyatomic aggregates?" As we shall show below, the endeavor to find such a process will lead us to formulate a new mechanism for a collective decay of two or more inner-shell vacancies.

Inner-shell-ionized states of atoms and molecules are very often higher in energy than their double ionization thresholds, a fact that gives rise to Auger process [3] in which one of the outer-shell electrons fills the inner-shell vacancy, while another outer-shell electron is ejected to continuum. Auger decay is mediated by electronic correlation within an atom (or a molecule) and typically occurs on the time scale of a few femtoseconds. Some inner-shell vacancies, e.g., inner-valence (IV) vacancies of rare gas atoms such as $\left(2 s^{-1}\right) \mathrm{Ne}^{+}$, are not energetic enough to give rise to Auger decay. In isolated systems, the low-energy IV- ionized states are bound to decay radiatively on the nanosecond time scale. A rather different scenario is realized whenever such a low-energy IV-ionized species is let to interact with an environment, for example, in a cluster. In such a case, the existence of the doubly ionized states with positive charges residing on two different cluster units leads to an interatomic (or intermolecular) decay process in which the recombination part of the two-electron transition takes part on one unit, whereas the ionization occurs on another one. This process, called interatomic (intermolecular) Coulombic decay (ICD) [5-7], is mediated by electronic correlation between two atoms (or molecules). In clusters of various sizes and compositions, ICD occurs on the time scale from hundreds of femtoseconds [8] down to several femtoseconds [9-12].

The common feature of Auger decay and ICD is that both processes represent a decay of a single vacancy. Thus, they are by no means specific to the multiply ionized systems, such as produced by an intense high-frequency FEL beam. For a decay process to occur exclusively in a multiply ionized system, it must actively involve more than a single vacancy. Here, we show that such a process in fact exists. Imagine an inner-shell vacancy state that is not energetic enough to decay by either Auger or ICD mechanisms. Such are, for example, $\mathrm{ns}^{-1}$ states of $\mathrm{Ar}^{+}, \mathrm{Kr}^{+}$and $\mathrm{Xe}^{+}$[13]. Consider further two such vacancy states, say, in a mixed $\mathrm{Kr}-X$ cluster, where $X$ is another atom or molecule. Neither of the vacancies can decay by electron emission because the energy provided by the $4 p \rightarrow 4 s$ recombination is not sufficient for $4 p$ ionization of either $\mathrm{Kr}^{+}$(as needed for Auger decay) or a neutral $\mathrm{Kr}$ (as needed for ICD). However, if two $4 s$-ionized kryptons recombine simultaneously, the released energy would be enough to ionize $X$ :

$$
\left(4 s^{-1}, 4 s^{-1}\right)\left(\mathrm{Kr}^{+}\right)_{2} X \rightarrow 2\left(4 p^{-1}\right) \mathrm{Kr}^{+}+X^{+}+e^{-} .
$$

A schematic representation of such a collective decay 
process that is possible only in a multiply inner-shellionized system is given in Fig. 1. Simple energy considerations imply that, in general, the collective decay occurs without facing a competition from the ICD if $1.5<\left(E_{\mathrm{iv}}-\right.$ $\left.E_{c}\right) / E_{\text {ion }}<2$, where $E_{\text {iv }}$ is the inner-valence ionization energy of the given species, $E_{c}$ is the energy of Coulombic repulsion between two singly ionized species (typically 3-4 eV at the equilibrium distances of neutral clusters), and $E_{\text {ion }}$ is the single ionization energy. Taking nonmetal hydrides [14], for example, one can easily make sure that the collective decay of the type of (1) is characteristic of multiply IV-ionized clusters of $\mathrm{HCl}, \mathrm{HBr}, \mathrm{HI}, \mathrm{H}_{2} \mathrm{~S}$, $\mathrm{H}_{2} \mathrm{Se}, \mathrm{H}_{2} \mathrm{Te}$, and $\mathrm{PH}_{3}$. Notably, the same conclusion holds for multiply IV-ionized clusters of small hydrocarbon molecules (e.g., of $\mathrm{C}_{2} \mathrm{H}_{6}, \mathrm{C}_{2} \mathrm{H}_{4}, \mathrm{C}_{2} \mathrm{H}_{2}$ for gerade IVionization).

The collective decay of Eq. (1) is mediated by electronic correlation between three atoms or molecules. Of course, the question arises: how realistic is the proposed decay mechanism? Indeed, competition to the collective decay can arise from the radiative decay of an isolated atom, as well as from the dissociative nuclear dynamics in the decaying doubly ionized cluster. Once formed, the doubly ionized cluster, e.g., of the $\left(\mathrm{Kr}^{+}\right)_{2} X$ type (see Fig. 1), will start disintegrating due to $\mathrm{Kr}^{+}-\mathrm{Kr}^{+}$repulsion. Depending on the magnitude of the decay rate at the initial cluster geometry and on its behavior at larger interatomic separations, the collective electronic transition can either take place or be suppressed by the cluster dissociation. Thus, estimation of the dependence of the decay rate on the cluster geometry is key to verification of the feasibility of the collective interatomic decay process.

Perhaps, the simplest way to estimate a rate of an electronic decay process is by means of the WignerWeisskopf theory that treats the initial and final states of the decay as single Slater determinants taking electronic

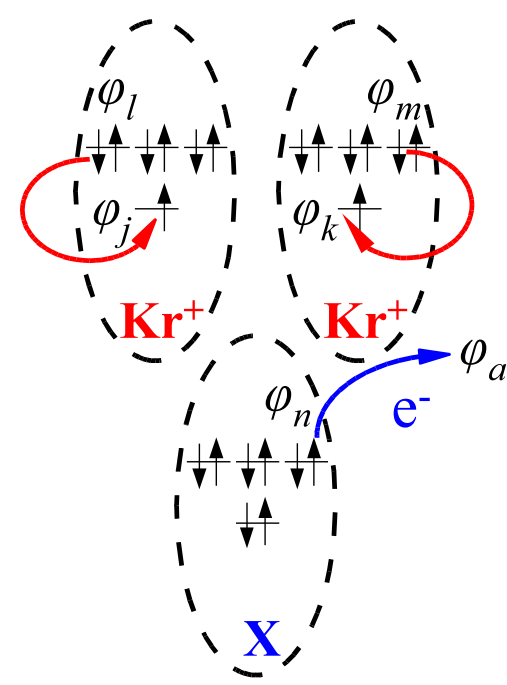

FIG. 1 (color online). Schematic representation of collective interatomic decay of two inner-shell vacancies. See Eq. (1) and the text for details. repulsion responsible for the transitions as a perturbation [10]. The collective decay of two inner-shell vacancies (see Fig. 1) is a three-electron transition mediated by twoelectron interaction. Thus, the process is forbidden in the first order perturbation theory. Going to the second-order perturbation theory, the Wigner-Weisskopf expression for the collective decay width can be written as

$$
\Gamma=2 \pi\left|\sum_{i} \frac{\langle f|\hat{V}| i\rangle\langle i|\hat{V}| 0\rangle}{E_{0}-E_{i}}\right|^{2} \delta\left(E_{f}-E_{0}\right), \quad \hat{V}=\sum_{p<q} \frac{e^{2}}{r_{p q}} .
$$

Here, $|0\rangle$ is the initial doubly inner-shell-ionized [(N-2)electron two-hole (2h)] state that can be derived from the $N$-electron Hartree-Fock (HF) ground state of the neutral system, $\left|\Phi_{0}^{\mathrm{HF}}\right\rangle$ by the application of the annihilation operators, $|0\rangle=\hat{c}_{j} \hat{c}_{k}\left|\Phi_{0}^{\mathrm{HF}}\right\rangle,|f\rangle$ is a final state of the three-hole-one-particle (3h1p) type, $|f\rangle=\hat{c}_{a}^{\dagger} \hat{c}_{l} \hat{c}_{m} \hat{c}_{n}\left|\Phi_{0}^{\mathrm{HF}}\right\rangle$, and $|i\rangle$ 's are the intermediate states. The $\delta$-function of the finalinitial energy difference in Eq. (2) reflects the conservation of energy in the course of the nonradiative transition.

Equation (2) suggests that the collective decay rate is formed by a superposition of interfering decay pathways, each of which is defined by an intermediate state, $|i\rangle$. Further analysis shows that such states can be either of $2 \mathrm{~h}$ or of $3 \mathrm{~h} 1 \mathrm{p}$ type. Consider, for example, $|i\rangle=$ $\hat{c}_{l} \hat{c}_{m}\left|\Phi_{0}^{\mathrm{HF}}\right\rangle$, where $\hat{c}_{l, m}$ correspond to the $\mathrm{Kr}$ outer-shell vacancies present in the final state, $|f\rangle$. Physically, this means that the tree-electron transition consists of virtual two-electron recombination on krypton cations followed by ionization of $X$ (see Fig. 1). The corresponding recombination matrix element entering Eq. (2) is

$$
\begin{aligned}
\langle i|V| 0\rangle= & \left\langle\Phi_{0}^{\mathrm{HF}} \hat{c}_{m}^{\dagger} \hat{c}_{l}^{\dagger}|\hat{V}| \hat{c}_{j} \hat{c}_{k} \Phi_{0}^{\mathrm{HF}}\right\rangle \\
= & \left\langle\varphi_{l}\left(\vec{r}_{1}\right) \varphi_{m}\left(\vec{r}_{2}\right)\left|1 / r_{12}\right| \varphi_{k}\left(\vec{r}_{1}\right) \varphi_{j}\left(\vec{r}_{2}\right)\right\rangle \\
& -\left\langle\varphi_{l}\left(\vec{r}_{1}\right) \varphi_{m}\left(\vec{r}_{2}\right)\left|1 / r_{12}\right| \varphi_{j}\left(\vec{r}_{1}\right) \varphi_{k}\left(\vec{r}_{2}\right)\right\rangle,
\end{aligned}
$$

where the electron repulsion matrix elements involve the outer shell $\left(\varphi_{l, m}\right)$ and the inner shell $\left(\varphi_{j, k}\right)$ of the $\mathrm{Kr}$ 's. Assuming that $\varphi_{j, l}$ are localized on the left $\mathrm{Kr}$, while $\varphi_{k, m}$ belong to the right $\mathrm{Kr}$ (see Fig. 1), we can obtain the dependence on the recombination matrix element on the interatomic distance, $R_{\mathrm{Kr}-\mathrm{Kr}}$, in the limit of large $\mathrm{Kr}-\mathrm{Kr}$ separation:

$$
\begin{aligned}
\langle i|V| 0\rangle \approx & -\left\langle\varphi_{l}\left(\vec{r}_{1}\right) \varphi_{m}\left(\vec{r}_{2}\right)\left|1 / r_{12}\right| \varphi_{j}\left(\vec{r}_{1}\right) \varphi_{k}\left(\vec{r}_{2}\right)\right\rangle \\
\approx & -\frac{1}{R_{\mathrm{Kr}-\mathrm{Kr}}^{3}}\left\langle\varphi_{l}\left(\vec{r}_{1}\right)\left|e \vec{r}_{1}\right| \varphi_{j}\left(\vec{r}_{1}\right)\right\rangle \\
& \cdot\left\langle\varphi_{m}\left(\vec{r}_{2}\right)\left|e \vec{r}_{2}\right| \varphi_{k}\left(\vec{r}_{2}\right)\right\rangle,
\end{aligned}
$$

where we have neglected the exchange contribution that decreases exponentially with $R_{\mathrm{Kr}-\mathrm{Kr}}$ and approximated the direct integral by the leading (dipole-dipole) term of the multipole expansion. The last expression suggests an appealing physical interpretation of the collective decay at large internuclear distances as a two-virtual-photon tran- 
sition with the two dipole matrix elements in Eq. (4) being regarded as two virtual photons that are "emitted" by the $\mathrm{Kr}^{+}$'s and "absorbed" by $X$. Within this simple picture, the collective interatomic decay is analogous to multiphoton transition, while Auger decay and ICD are analogous to a single-photon transition.

Proceeding with the large- $R$ analysis of the ionization matrix element, $\langle f|\hat{V}| i\rangle$, we find that at large $\mathrm{Kr}_{2}-X$ separations it decreases as $1 / R_{\mathrm{Kr}_{2}-X}^{2}$. As a result, the contribution of the two-electron recombination-ionization pathway to the Wigner-Weisskopf expression for the decay width depends on the cluster geometry as $1 / R_{\mathrm{Kr}-\mathrm{Kr}_{\mathrm{r}}}^{6} R_{\mathrm{Kr}_{2}-X}^{4}$. Since the decomposition of the $\left(\mathrm{Kr}^{+}\right)_{2} X$ cluster along the $\mathrm{Kr}^{+}-\mathrm{Kr}^{+}$coordinate automatically means elongation of the $\mathrm{Kr}_{2}-X$ distance as well, the power law exponents are effectively summed, resulting in the $1 / R_{\mathrm{Kr}-\mathrm{Kr}}^{10}$ dependence.
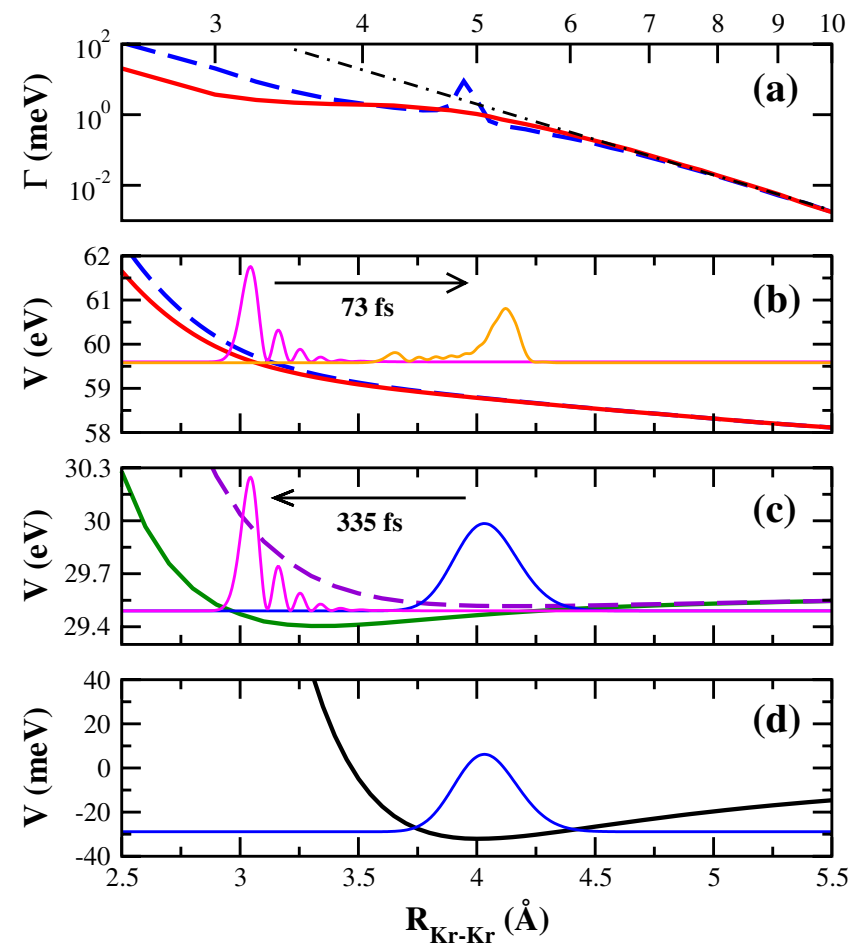

FIG. 2 (color online). (a) Collective decay widths of $\left(4 s^{-1}\right.$, $\left.4 s^{-1}\right)\left(\mathrm{Kr}^{+}\right)_{2} \mathrm{Ar}$ as functions of $R_{\mathrm{Kr}-\mathrm{Kr}}$ at $R_{\mathrm{Kr}_{2}-\mathrm{Ar}}^{\mathrm{eq}}=3.3 \AA$. Solid line - triplet, dashed line-singlet, dashed-dotted line- $1 / R^{10} \mathrm{fit}$ (please, note the doubly logarithmic scale). (b) PESs of $\left(4 s^{-1}\right.$, $\left.4 s^{-1}\right)\left(\mathrm{Kr}^{+}\right)_{2}$ Ar states, cut at $R_{\mathrm{Kr}_{2}-\mathrm{Ar}}^{\mathrm{eq}}=3.3 \AA$. Solid line-triplet state, dashed line-singlet state. Shown also is the vibrational wave packet promoted from the $\left(4 s \sigma_{u}^{-1}\right)$ state of $\left(\mathrm{Kr}_{2}\right)^{+} \mathrm{Ar}$ [see panel (c)] at the moment of closest approach of the two kryptons, $t_{\min }=335 \mathrm{fs}$, and $73 \mathrm{fs}$ later. Direction of motion of the wave packet is shown by an arrow. (c) PESs of $\left(4 s \sigma_{g, u}^{-1}\right)\left(\mathrm{Kr}_{2}\right)^{+} \mathrm{Ar}$ states, cut at $R_{\mathrm{Kr}_{2}-\mathrm{Ar}}^{\mathrm{eq}}=3.3 \AA$. Solid line-ungerade state, dashed line-gerade state. Shown also is the vibrational wave packet promoted from the ground state [see panel (d)] at $t=0$ (right) and at $t_{\min }$ (left). Direction of motion of the wave packet is shown by an arrow. (d) PES of the neutral $\left(\mathrm{Kr}_{2}\right) \mathrm{Ar}$, cut at $R_{\mathrm{Kr}_{2}-\mathrm{Ar}}^{\mathrm{eq}}=3.3 \AA$. Shown also is the vibrational ground state.
A detailed analysis shows that this type of power law is characteristic of all the possible decay pathways.

While the Wigner-Weisskopf expression for the decay rate (2) can be evaluated numerically, the inaccuracy of the lowest-order perturbation theory results can easily reach a factor of two (see, e.g., Ref. [8]). Far more accurate decay widths can be obtained by the Fano-ADC technique recently generalized for the decay of doubly ionized [15] systems. Fano-ADC approach for electronic decay widths is an $\mathcal{L}^{2}$ method that is based on the Fano expression [16] for $\Gamma$ through the matrix element of the full Hamiltonian $(\hat{H})$ between the boundlike $(\Phi)$ and the continuumlike $\left(\chi \alpha, \varepsilon_{\alpha}\right)$ components of the wave function at the energy of the resonance $\left(E_{r}\right)$,

$$
\Gamma=2 \pi \sum_{\alpha=1}^{N_{c}}\left|\left\langle\Phi\left|\hat{H}-E_{r}\right| \chi_{\alpha, \varepsilon_{\alpha}}\right\rangle\right|^{2},
$$

where the summation is over $N_{c}$ decay channels and $\varepsilon_{\alpha}$ is the kinetic energy of the emitted electron for the $\alpha$ 's decay channel. The $(N-2)$-electron wave functions $\Phi$ and $\chi_{\alpha, \varepsilon_{\alpha}}$ are obtained using the ab initio method known as extended second-order algebraic diagrammatic construction (ADC) [17]. Finally, the renormalization of the Hamiltonian matrix elements obtained with $\mathcal{L}^{2}$ wave functions is achieved by Stieltjes imaging procedure [18].

Here, we use the Fano-ADC method of Ref. [15] to calculate the collective interatomic decay widths for $\left(4 s^{-1}, 4 s^{-1}\right)\left(\mathrm{Kr}^{+}\right)_{2} \mathrm{Ar}$ cluster, i.e., for $X=\operatorname{Ar}$ [see Eq. (1)]. The results for $\Gamma$ as a function of the $\mathrm{Kr}-\mathrm{Kr}$ distance (at $R_{\mathrm{Kr}_{2}-\mathrm{Ar}}^{\mathrm{eq}}=3.3 \AA$ ) are shown in Fig. 2(a). At the equilibrium $\mathrm{Kr}-\mathrm{Kr}$ distance $\left(R_{\mathrm{Kr}_{2}}^{\mathrm{eq}}=4.0 \AA\right)$, the collective decay width reaches $1.9 \mathrm{meV}$ for triplet and $2.0 \mathrm{meV}$ for singlet doubly ionized states, which corresponds to life times around $300 \mathrm{fs}$. The predicted life time is 5 orders of magnitude shorter than the one of the radiative decay [19] which means that the process is not suppressed by photon emission. The enhancement of the singlet width around $R=4.9 \AA$ is due to the interaction with the singlet PES of the $\left(4 s^{-1}, 4 p^{-1}\right) \mathrm{Kr}^{2+} \mathrm{Kr}$ type. At $R_{\mathrm{Kr}-\mathrm{Kr}} 6 \AA$, the collective decay width shows the predicted $1 / R^{10}$ behavior [see Fig. 2(a)].

Having obtained quantitative predictions for the collective decay rate (see Fig. 2), we are in a position to consider the competition between the electronic decay process and the dissociation of $\left(4 s^{-1}, 4 s^{-1}\right)\left(\mathrm{Kr}^{+}\right){ }_{2} \mathrm{Ar}$. This can be done conveniently within the Born-Oppenheimer (BO) picture by following the evolution of the vibrational wave packet on the initial (neutral), intermediate (singly and doubly ionized), and final (triply ionized) potential energy surfaces (PESs). Of course, we shall mainly consider the $R_{\mathrm{Kr}-\mathrm{Kr}}$ degree of freedom as the one along which the dissociation occurs. The relevant cuts of the $\mathrm{Kr}_{2} \mathrm{Ar}$ PESs at the symmetric geometry with $R_{\mathrm{Kr}_{2}-\mathrm{Ar}}$ frozen at its equilibrium value are shown in Fig. 2. We assume that in the beginning the cluster is in its ground vibrational state. 
At some later time, $4 s$ photoionization occurs on the $\mathrm{Kr}$ atoms due to the interaction with the high-intensity FEL beam. The double photoionization can occur either directly by a "vertical," i.e., instantaneous transition to the $\left(4 s^{-1}\right.$, $\left.4 s^{-1}\right)\left(\mathrm{Kr}^{+}\right)_{2} \mathrm{Ar}$ PES or in two stages, bringing the cluster first onto a $\left(\mathrm{Kr}_{2}\right)^{+}$Ar PES and only later (by another vertical transition) to a doubly ionized PES (see Fig. 2). The important difference between the one-step and the two-step processes is that the former creates a vibrational wave packet centered around the equilibrium distance of the neutral $\left(R_{\mathrm{Kr}-\mathrm{Kr}}=4.0 \AA\right)$, while in the latter process, the wave packet created on the singly ionized PES can evolve before being promoted to the repulsive decaying state. If at the first stage of the sequential process the $(4 s) \sigma_{u}$ orbital of $\mathrm{Kr}_{2}$ is ionized, the resulting vibrational motion can explore the region of much smaller $\mathrm{Kr}-\mathrm{Kr}$ distances than in the case of the one-step double ionization. Thus, the two-step ionization process, in particular, the one proceeding via $(4 s) \sigma_{u}$ ionization, leads the system to the region of much higher collective decay widths than the one-step double ionization and is expected to give a higher yield of the electronic decay. In order to quantify these assessments, we have performed a series of nuclear dynamics simulations on the PESs cuts shown in Fig. 2, i.e., assuming that Ar atom is fixed in the course of photoionization and decay. Our results show that the collective interatomic decay yields in the case of one-step double photoionization are $31 \%$ and $38 \%$ (for triplet and singlet doubly ionized states, respectively), whereas for the two-step ionization into the singlet state, the electronic decay yield can reach $65 \%$. In the case of $\mathrm{Kr}_{2} X$ systems, however, the actual yield will be affected not only by the ionization mode, but also by the duration of the FEL pulse. Indeed, the currently available pulses with duration of up to $100 \mathrm{fs}$ are shorter than the time it takes the two Kr's to reach the point of their closest approach [see Fig. 2(c)]. The nonradiative $\mathrm{Kr}^{+}$ionization according to $\left(4 s^{-1}, 4 s^{-1}\right)\left(\mathrm{Kr}^{+}\right)_{2} X \rightarrow\left(4 p^{-1}\right) \mathrm{Kr}^{+}+\left(4 p^{-2}\right) \mathrm{Kr}^{2+}+$ $X+e^{-}$that is energetically allowed only at $R \geq 5.4 \AA$ has been verified not to play a significant role in any of the above ionization-decay scenarios.

In conclusion, we have predicted a new electronic decay process-collective interatomic decay of inner-shell vacancies. The new decay mechanism is relevant for the case of cluster interaction with intense high-frequency radiation that is expected to produce multiple inner-shell vacancies. Perturbation theory analysis shows that the collective decay process can be regarded as a multivirtual-photon transition, an analog to multiphoton ionization. Using a combination of $a b$ initio calculations of the rate of the collective decay and nuclear dynamics simulations, we have shown that in the case of the decay of two inner-shell vacancies in the $\mathrm{Kr}_{2} \mathrm{Ar}$ van der Waals cluster, the nonradiative process is orders of magnitude faster than the decay of a single vacancy by photon emission and can compete successfully with the disintegration of the doubly ionized cluster by Coulombic explosion. The fingerprint of the new decay process that should allow its experimental observation is the kinetic energy distribution of the produced electrons [see Eq. (1)] that in the case of $\mathrm{Kr}_{2} \mathrm{Ar}$ would spread between 3 and $4 \mathrm{eV}$ for the double ionization scenarios considered above. The collective interatomic (intermolecular) decay mechanism proposed here appears to be quite general, being relevant far beyond the heavier rare gas clusters, e.g., mixed $\mathrm{Kr}$-Ar ones. For example, a wide variety of nonmetal hydrides having $\mathrm{Ar}$, $\mathrm{Kr}$, or $\mathrm{Xe}$ as their united atom limit are expected to exhibit the collective decay (and not ICD or Auger decay) in their multiply IV-ionized clusters. Most importantly, the predicted decay phenomenon is expected to occur unhindered by other electronic processes in clusters of small hydrocarbons, i.e., it can clearly be relevant for the mechanisms of radiation damage in FEL interaction with organic compounds.

V. A. would like to acknowledge the financial support of the Max Planck Society. P. K. acknowledges the financial support from the Grant No. GAČR 202/09/0786 of the Czech Science Foundation and from Záměr MSM0021620860. The authors would like to thank Dr. H. Scherrer for his help with the computer use at MPI PKS, Dresden.

*vitali@mpipks-dresden.mpg.de

${ }^{\dagger}$ kolorenc@mbox.troja.mff.cuni.cz

[1] J. Feldhaus et al., J. Phys. B 38, S799 (2005).

[2] U. Saalmann and J. M. Rost, Phys. Rev. Lett. 89, 143401 (2002).

[3] W. Mehlhorn in Atomic Inner-Shell Physics, edited by B. Crasemann (Plenum, New York, 1985).

[4] R. Neutze et al., Nature (London) 406, 752 (2000).

[5] L. S. Cederbaum, J. Zobeley, and F. Tarantelli, Phys. Rev. Lett. 79, 4778 (1997).

[6] S. Marburger et al., Phys. Rev. Lett. 90, 203401 (2003).

[7] T. Jahnke et al., Phys. Rev. Lett. 93, 163401 (2004).

[8] V. Averbukh and L. S. Cederbaum, J. Chem. Phys. 123, 204107 (2005); 125, 094107 (2006).

[9] G. Öhrwall et al., Phys. Rev. Lett. 93, 173401 (2004).

[10] R. Santra, J. Zobeley, and L. S. Cederbaum, Phys. Rev. B 64, 245104 (2001).

[11] N. Vaval and L.S. Cederbaum, J. Chem. Phys. 126, 164110 (2007).

[12] V. Averbukh and L.S. Cederbaum, Phys. Rev. Lett. 96, 053401 (2006).

[13] Here, ICD can occur only from satellite states, see P. Lablanquie et al., J. Chem. Phys. 127, 154323 (2007).

[14] A. W. Potts and W. C. Price, Proc. R. Soc. A 326, 181 (1972).

[15] P. Kolorenč et al., J. Chem. Phys. 129, 244102 (2008).

[16] U. Fano, Phys. Rev. 124, 1866 (1961).

[17] J. Schirmer and A. Barth, Z. Phys. A 317, 267 (1984).

[18] P. Langhoff, Chem. Phys. Lett. 22, 60 (1973).

[19] S. Lauer et al., J. Phys. B 32, 2015 (1999). 